\title{
NFI gene mutations are the major molecular event in neurofibromatosis-noonan syndrome
}

\begin{abstract}
Neurofibromatosis-Noonan syndrome is a rare autosomal dominant disorder which combines neurofibromatosis type 1 (NF1) features with Noonan syndrome. Noonan syndrome (NS) and the clinically overlapping disorders and Neurofibromatosis-Noonan syndrome (NFNS) share the some common clinical features. It is now known that all these disorders are caused by mutations in components of the RAS-MAPK signaling pathway which is important in tumorigenesis. NF1 gene mutations are reported in the majority of these patients. There are some data in the literature about the NF1 mutant allele which can lead to manifestations of Noonan syndrome. We have studied four NFNS cases which all fit the NFNS criteria. We evaluated these patients one with Watson syndrome (WS) and the other one with Rhabdomyosarcoma. Although WS and NFNS were described as distinct disorders, detailed clinical examination of these families revealed that not only pulmonic stenosis, borderline intelligence, and multiple cafe'-au-lait spots, but also multiple Lisch nodules, neurofibromas in one third of patients, and short stature were present. The only distinction between WS and NFNS would be that NFNS patients show a more classical phenotype of both NS and NF, whereas WS patients show only a mild expression of NF. Recently, there is increasing evidence for WS and NFNS being allelic to NF1 in the majority of patients. We analyzed 4 NFNS patients by PCR based techniques. Genomic DNA was extracted from peripheral blood samples. PCR was performed with intronic primers for all exons of the NF1. DNA samples were sequenced to detect variations in each exon. This study supports that the major gene causing NFNS is NF1. Therefore inclusion of NF1 in the genetic screening of patients with clinically suspected NS, preferentially when café-au-lait spots are present. As a result, the present study provides the molecular evidence of the role of NF1 mutations in NFNS.
\end{abstract}

Keywords: neurofibromatosis type 1,noonan syndrome;neurofibromatosis - noonan syndrome, $\mathrm{nfl}$ gene mutations
Volume 6 Issue 6 - 2017

\section{Sharafi P,' Anlar B, ${ }^{2}$ Ayter $S^{\prime}$}

'Department of Medical Biology and Genetics, TOBB University of Economics and Technology, Turkey

${ }^{2}$ Department of Pediatrics, Pediatric Neurology, Hacettepe University, Turkey

Correspondence: Șükriye AYTER, TOBB University of Economics and Technology, Faculty of Medicine, Ankara, Turkey, Tel 903/22924000, Fax 9.03123E+ | I, Email sayter@etu.edu.tr

Received: April 05, 2017 | Published: May 24, 2017
Abbreviations: NF1, neurofibromatosis type 1; NS, noonan syndrome; NFNS, neurofibromatosis-noonan syndrome; WS, watson syndrome; LOH, loss of heterozygosity; PCR, polymerase chain reaction; CLS, café-au-lait spots; AF, axillary freckling; IF, inguinal freckling; LN, lisch nodules; SD, skeletal dysplasia; RMS, rhabdomyosarcoma

\section{Introduction}

The Neurofibromatosis type 1 disease (NF1) (OMIM:162200), which is known also as von Recklinghausen's Disease, is an autosomal dominant disorder with an estimated frequency of 1 in 2500 to 3000 live births. ${ }^{1}$ The gene of neurofibromatosis type 1 is located at chromosome $17 \mathrm{q} 11.2 .{ }^{2}$ Approximately $50 \%$ of all affected individuals carry de novo mutations. ${ }^{3,4}$ Typical appearances are café-au lait spots, peripheral neurofibromas, Lisch nodules, axillary and inguinal freckling, malignant peripheral nerve sheath tumors (MPNST) (Neurofibromas as a benign form and Neurofibrosarcomas as a malignant forms) and other malignancies such as intracranial astrocytomas, gastrointestinal stromal tumors, pheochromocytomas, and juvenile monocytic leukemia. ${ }^{2,5}$ Endocrine symptoms, neurological and ophthalmological problems are the other manifestations that appear less frequently. ${ }^{6}$

Noonan's syndrome (NS) (OMIM: 605275) is also an autosomal dominant disorder with massive heterogeneity in clinical and genetic features same as NF1. The incidence of NS is 1 in 1000 to 2500 live births. $^{7}$ The clinical feature of NS is short stature, congenital heart defects, unusual pectus deformity, and typical facial features, such as hypertelorism, ptosis, downslanting palpebral fissures, low-set posteriorly rotated ears, and a broad forehead. Additional associated features include neonatal failure to thrive, bleeding abnormalities, mild intellectual disability, multiple skeletal defects, and various skin manifestations, for example, cafe'-au-lait $\operatorname{spots}^{2,8,9}$ with both hematopoietic malignancies and malignant solid Tumors. ${ }^{10}$ Nine genes involved in the RAS-MAPK pathway are known to be associated with $70-85 \%$ of patients with NS or NS-like conditions. These genes are PTPN11 (responsible gene for $50 \%$ of patients), SOS1, KRAS, NRAS, BRAF, RAF1, MAP2K1, CBL, and SHOC2. ${ }^{11}$

A atypical clinical association of these two disorders, named Neurofibromatosis-Noonan syndrome (NFNS) MIM 601321 is first noted in 1985 by Allanson and colleagues. ${ }^{12}$ They described subjects with features of both NF1 and NS ${ }^{13}$. Though it has been long speculated for many years that whether NFNS is a different form of either NF1 or NS or they are distinct disorders, ${ }^{14}$ approximately the $13 \%$ of patients with NF1 display the phenotypic features of NS. ${ }^{15}$ This phenotype in such cases is most probably produced by genes that are very close to the NF1 locus or by variable expression of NF1 gene. ${ }^{16}$ In this study the aim is to support that NF1 gene mutations are the major causing NFNS.

\section{Case Presentation}

The clinical and genetic analyses were performed according to the guidelines in the Declaration of Helsinki and approved by the ethical committee of Hacettepe University, Turkey. This study included four individuals from unrelated families, presenting clinical features of both NF1 and NS. To diagnose the NF1, the NIH-consensus statement 
was used, ${ }^{6}$ and to diagnose NS a checklist of phenotypic features was used. ${ }^{17}$

\section{Patients}

This study included four individuals from unrelated families, presenting features of both NF1 and NS.

Case 1: An 11-years-old girl with familial history of NF1. She presented NS symptoms together with café-au-lait spots distributed throughout the body together with axillary frecklings and Lisch nodules.

Case 2: A 16-years-old boy with familial history of NF1. The Physical examinations and ultrasonography results showed the presence of Noonan and Watson syndromes (pulmonary valvular stenosis, relative macrocephaly, and short stature) together with café-au-lait spots and bone dysplasia.
Case 3: A 6-years-old boy without familial history. The presence of café-au-lait spots with NF1 gene mutation and symptoms of NS was detected.

Case 4: A 12-month-old boy without familial history with the complaints of difficulty urinating and abdominal distention. $\mathrm{He}$ had café-au-lait spots throughout the body together with inguinal freckling. The patient's facial appearance and the clinical features was distinct for NS. ${ }^{18}$

\section{DNA analysis}

Genomic DNA was extracted from peripheral blood leukocytes from affected and healthy individuals according to standard procedures. Genomic DNA samples were PCR amplified with intronic primers for all exons and sequenced in both forward and reverse direction for all exons and flanking intron sequences of NF1. NF1 mutations were detected in 3 of patients with Noonan syndrome (Table 1).

Table I Summary of Noonan patients with and without mutations in NFI gene

\begin{tabular}{|c|c|c|c|c|c|c|}
\hline No & Pt. No & & Exon & Nucleotide Change & Mutation & Clinical Symptoms \\
\hline $\mathrm{I}$ & 45 & familial & - & not detected & - & CLS, AF, LN, Noonan \\
\hline 2 & 124 & familial & 16 & $c 285 \mathrm{I}-\mathrm{I} 6 \mathrm{~T}>\mathrm{C}$ & nonsense & CLS, SD, Watson, Noonan \\
\hline 3 & 329 & sporadic & 26 & c. $4397 \mathrm{C}>\mathrm{T}$ & Missense & CLS, Noonan \\
\hline 4 & 385 & sporadic & Whole gene & Large Deletion & Deletions & CLS, AF, IF, Rhabdomyosarcoma, Noonan \\
\hline
\end{tabular}

CLS: Café-au-lait Spots ;AL:Axillary Freckling; IF: Inguinal Freckling; LN: Lisch Nodules; SD: Skeletal Dysplasia

\section{Discussion}

In the literature it has mentioned that several gene mutations are associated with NS. However, there have been several reports supporting the existence of NF1 gene mutations in the NFNS cases. In the first report, 3-bp deletion was found in exon 17 in a two generation family with NFNS. ${ }^{19}$ In another case, Baralle et al., ${ }^{19}$ found a 2-bp insertion in exon 23.2 and a 3-bp deletion in exon 25 in NFNS patients. Bahuau et al., ${ }^{2}$ also reported on a three-generation NF-NS family who has a nonsense mutation in exon 16. Moreover, Yimenicioglu et al., ${ }^{20}$ presented another case report with a point mutation c. $7549 \mathrm{C}>\mathrm{T}$ in exon 51 in NF1 gene for the typical phenotype of both NFNS.

Although NF1 gene mutations have been reported in NF-NS patients, there is still no genotype-phenotype correlation. The NFNS phenotype is heterogeneous at both the clinical and the molecular level. Several different causes may be involved in simultaneous occurrence of NF1 and NS. In our study we also detected NF1 gene mutations in three out of four NFNS cases which support that NF1 gene mutations seems like the major cause in NFNS cases. Therefore inclusion of NF1 in the genetic screening of patients with clinically suspected NS are important, preferentially when cafe'-au-lait spots are present. To confirm these associations and to give a better genetic counseling for these patients, further studies are required.

\section{Acknowledgements}

In Memory of Prof. Dr. Murat Derbent.

\section{Conflicts of interest}

None.

\section{Funding}

None.

\section{References}

1. Matsui I, Tanimura M, Kobayashi N, et al. Neurofibromatosis type 1 and childhood cancer. Cancer. 1993;72(9):2746-2754.
2. Bahuau M, Houdayer C, Assouline B, et al. Novel recurrent nonsense mutation causing neurofibromatosis type 1 (NF1) in a family segregating both NF1 and Noonan syndrome. Am J Med Genet. 1998;75(3):265-272.

3. Brosius S. A history of von Recklinghausen's NF1. J Hist Neurosci. 2010;19(4):333-348

4. Li Y, O'Connell P, Breidenbach HH, et al. Genomic organization of the neurofibromatosis 1 gene (NF1). Genomics. 1995;25(1):9-18.

5. Theos A, Korf BR, American College of P, et al. Pathophysiology of neurofibromatosis type 1. Ann Intern Med. 2006;144:842-849.

6. Ferner RE, Huson SM, Thomas N, et al. Guidelines for the diagnosis and management of individuals with neurofibromatosis $1 . \mathrm{JMed}$ Genet. 2007;44(2):81-88.

7. Roberts AE, Allanson JE, Tartaglia M, et al. Noonan syndrome. Lancet. 2013;381:333-342.

8. Allanson JE. Noonan syndrome. J Med Genet. 1987;24:9-13.

9. van der Burgt I. Noonan syndrome. Orphanet J Rare Dis. 2007;2:4.

10. Smpokou P, Zand DJ, Rosenbaum KN, et al. Malignancy in Noonan syndrome and related disorders. Clin Genet. 2015;88(6):516-522.

11. Ekvall S, Sjors K, Jonzon A, et al. Novel association of neurofibromatosis type 1-causing mutations in families with neurofibromatosis-Noonan syndrome. Am J Med Genet A. 2014; 164A(3):579-587.

12. Allanson JE, Hall JG, Van Allen MI. Noonan phenotype associated with neurofibromatosis. Am J Med Genet. 1985;21(3):457-462.

13. Opitz JM, Weaver DD. The neurofibromatosis-Noonan syndrome. Am J Med Genet . 1985;21(3): 477-490.

14. De Luca A, Bottillo I, Sarkozy A, et al. NF1 gene mutations represent the major molecular event underlying neurofibromatosis-Noonan syndrome. Am J Hum Genet. 2005;77(6):1092-1101.

15. Colman SD, Rasmussen SA, Ho VT, et al. Somatic mosaicism in a patient with neurofibromatosis type 1. Am J Hum Genet. 1996;58(3):484-490.

16. Stern HJ, Saal HM, Lee JS, et al. Clinical variability of type 1 neurofibromatosis: is there a neurofibromatosis-Noonan syndrome? $J$ Med Genet. 1992;29(3):184-187. 
17. Nystrom AM, Ekvall S, Berglund E, et al. Noonan and cardio-faciocutaneous syndromes: two clinically and genetically overlapping disorders. J Med Genet. 2008;45(8):500-506.

18. Agras PI, Baskin E, Sakallioglu AE, et al. Neurofibromatosis--Noonan's syndrome with associated rhabdomyosarcoma of the urinary bladder in an infant: case report. J Child Neurol. 2003;18(1):68-72.
19. Baralle D, Mattocks C, Kalidas K, et al. Different mutations in the NF1 gene are associated with Neurofibromatosis-Noonan syndrome (NFNS). Am J Med Genet A. 2003;119A(1):1-8.

20. Yimenicioglu S, Yakut A, Karaer K, et al. A new nonsense mutation in the NF1 gene with neurofibromatosis-Noonan syndrome phenotype. Childs Nerv Syst. 2012;28(12):2181-2183. 